\title{
FIXED POINT THEORY FOR MÖNCH-TYPE MAPS DEFINED ON CLOSED SUBSETS OF FRÉCHET SPACES: THE PROJECTIVE LIMIT APPROACH
}

\author{
RAVI P. AGARWAL, JEWGENI H. DSHALALOW, AND DONAL O'REGAN
}

Received 17 May 2005

New Leray-Schauder alternatives are presented for Mönch-type maps defined between Fréchet spaces. The proof relies on viewing a Fréchet space as the projective limit of a sequence of Banach spaces.

\section{Introduction}

This paper presents new Leray-Schauder alternatives for Mönch-type maps defined between Fréchet spaces. Two approaches $[1,2,3,6,7]$ have recently been presented in the literature both of which are based on the fact that a Fréchet space can be viewed as a projective limit of a sequence of Banach spaces $\left\{E_{n}\right\}_{n \in \mathbb{N}}$ (here $\mathbb{N}=\{1,2, \ldots\}$ ). Both approaches are based on constructing maps $F_{n}$ defined on subsets of $E_{n}$ whose fixed points converge to a fixed point of the original operator $F$. Both approaches have advantages and disadvantages over the other [1] and in this paper, we combine the advantages of both approaches to present very general fixed point results. Our theory in particular extends and improves the theory in [3] (in [3], the single-valued case was discussed).

Finally in this section, we gather together some definitions and a fixed point result which will be needed in Section 2 .

Now, let $I$ be a directed set with order $\leq$ and let $\left\{E_{\alpha}\right\}_{\alpha \in I}$ be a family of locally convex spaces. For each $\alpha \in I, \beta \in I$ for which $\alpha \leq \beta$, let $\pi_{\alpha, \beta}: E_{\beta} \rightarrow E_{\alpha}$ be a continuous map. Then the set

$$
\left\{x=\left(x_{\alpha}\right) \in \prod_{\alpha \in I} E_{\alpha}: x_{\alpha}=\pi_{\alpha, \beta}\left(x_{\beta}\right) \forall \alpha, \beta \in I, \alpha \leq \beta\right\}
$$

is a closed subset of $\prod_{\alpha \in I} E_{\alpha}$ and is called the projective limit of $\left\{E_{\alpha}\right\}_{\alpha \in I}$ and is denoted by $\lim _{\leftarrow} E_{\alpha}$ (or $\lim _{\leftarrow}\left\{E_{\alpha}, \pi_{\alpha, \beta}\right\}$ or the generalized intersection [4, page 439] $\bigcap_{\alpha \in I} E_{\alpha}$ ).

Next, we recall a fixed point result from the literature [9] which we will use in Section 2.

Theorem 1.1. Let $K$ be a closed convex subset of a Banach space $X, U$ a relatively open subset of $K, x_{0} \in U$, and suppose that $F: \bar{U} \rightarrow C K(K)$ is an upper semicontinuous map (here $C K(K)$ denotes the family of nonempty convex compact subsets of $K$ ). Also assume 
that the following conditions hold:

$$
\begin{aligned}
& M \subseteq \bar{U}, \quad M \subseteq \operatorname{co}\left(\left\{x_{0}\right\} \cup F(M)\right) \quad \text { with } \bar{M}=\bar{C}, \\
& C \subseteq M \quad \text { countable, implies } \bar{M} \text { is compact, } \\
& x \notin(1-\lambda)\left\{x_{0}\right\}+\lambda F x \quad \text { for } x \in \bar{U} \backslash U, \lambda \in(0,1) .
\end{aligned}
$$

Then there exist a compact set $\sum$ of $\bar{U}$ and an $x \in \sum$ with $x \in F x$.

Remark 1.2. In [9], we see that we could take $\sum$ to be

$$
\left\{y \in \bar{U}: y \in(1-\lambda)\left\{x_{0}\right\}+\lambda F y \text { for some } \lambda \in[0,1]\right\}
$$

We did not show that $\sum$ is compact in [9] but this is easy to see as we will now show. First, notice that $\sum$ is closed since $F$ is upper semicontinuous. Now let $\left\{y_{n}\right\}_{1}^{\infty}$ be a sequence in $\sum$. Then there exists $\left\{t_{n}\right\}_{1}^{\infty}$ in $[0,1]$ with $y_{n} \in\left(1-t_{n}\right)\left\{x_{0}\right\}+t_{n} F y_{n}$ for $n \in \mathbb{N}=\{1,2, \ldots\}$. Without loss of generality, assume that $t_{n} \rightarrow t \in[0,1]$. Let $C=\left\{y_{n}\right\}_{1}^{\infty}$. Notice that $C$ is countable and $C \subseteq \operatorname{co}\left(\left\{x_{0}\right\} \cup F(C)\right)$. Now (1.2) with $M=C$ guarantees that $\bar{C}$ is compact (so sequentially compact). Thus there exist a subsequence $N_{1}$ of $\mathbb{N}$ and a $y \in \bar{C}$ with $y_{n} \rightarrow y$ as $n \rightarrow \infty$ in $N_{1}$. This together with $y_{n} \in\left(1-t_{n}\right)\left\{x_{0}\right\}+t_{n} F y_{n}$ and the upper semicontinuity of $F$ guarantees that $y \in(1-t)\left\{x_{0}\right\}+t F y$, so $y \in \bar{\sum}=\sum$. Consequently, $\sum$ is sequentially compact and hence compact. In fact, one could also of course take $\sum$ to be

$$
\{y \in \bar{U}: y \in F y\}
$$

for the compact set in Theorem 1.1.

\section{Projective limit approach}

Let $E=\left(E,\left\{|\cdot|_{n}\right\}_{n \in \mathbb{N}}\right)$ be a Fréchet space with the topology generated by a family of seminorms $\left\{|\cdot|_{n}: n \in \mathbb{N}\right\}$. We assume that the family of seminorms satisfies

$$
|x|_{1} \leq|x|_{2} \leq|x|_{3} \leq \cdots \quad \text { for every } x \in E \text {. }
$$

To $E$, we associate a sequence of Banach spaces $\left\{\left(\mathbf{E}_{n},|\cdot|_{n}\right)\right\}$ described as follows. For every $n \in \mathbb{N}$, we consider the equivalence relation $\sim_{n}$ defined by

$$
x \sim_{n} y \quad \text { iff }|x-y|_{n}=0 .
$$

We denote by $\mathbf{E}^{n}=\left(E / \sim_{n},|\cdot|_{n}\right)$ the quotient space, and by $\left(\mathbf{E}_{n},|\cdot|_{n}\right)$ the completion of $\mathbf{E}^{n}$ with respect to $|\cdot|_{n}$ (the norm on $\mathbf{E}^{n}$ induced by $|\cdot|_{n}$ and its extension to $\mathbf{E}_{n}$ are still denoted by $\left.|\cdot|_{n}\right)$. This construction defines a continuous map $\mu_{n}: E \rightarrow \mathbf{E}_{n}$. Now since (2.1) is satisfied, the seminorm $|\cdot|_{n}$ induces a seminorm on $\mathbf{E}_{m}$ for every $m \geq n$ (again this seminorm is denoted by $|\cdot|_{n}$ ). Also (2.2) defines an equivalence relation on $\mathbf{E}_{m}$ from which we obtain a continuous map $\mu_{n, m}: \mathbf{E}_{m} \rightarrow \mathbf{E}_{n}$ since $\mathbf{E}_{m} / \sim_{n}$ can be regarded as 
a subset of $\mathbf{E}_{n}$. We now assume that the following condition holds:

$$
\begin{aligned}
& \text { for each } n \in \mathbb{N} \text {, there exist a Banach space }\left(E_{n},|\cdot|_{n}\right) \\
& \text { and an isomorphism (between normed spaces) } j_{n}: \mathbf{E}_{n} \longrightarrow E_{n} \text {. }
\end{aligned}
$$

Remark 2.1. (i) For convenience, the norm on $E_{n}$ is denoted by $|\cdot|_{n}$.

(ii) In our applications, $\mathbf{E}_{n}=\mathbf{E}^{n}$ for each $n \in \mathbb{N}$.

(iii) Note that if $x \in \mathbf{E}_{n}$ (or $\mathbf{E}^{n}$ ), then $x \in E$. However if $x \in E_{n}$, then $x$ is not necessarily in $E$ and in fact $E_{n}$ is easier to use in applications as we will see in Theorem 2.3 (even though $E_{n}$ is isomorphic to $\mathbf{E}_{n}$ ).

Finally, we assume that

$$
E_{1} \supseteq E_{2} \supseteq \cdots \quad \text { and for each } n \in \mathbb{N}, \quad|x|_{n} \leq|x|_{n+1} \quad \forall x \in E_{n+1} .
$$

Let $\lim _{\leftarrow} E_{n}$ (or $\bigcap_{1}^{\infty} E_{n}$, where $\bigcap_{1}^{\infty}$ is the generalized intersection [4]) denote the projective limit of $\left\{E_{n}\right\}_{n \in \mathbb{N}}$ (note that $\pi_{n, m}=j_{n} \mu_{n, m} j_{m}^{-1}: E_{m} \rightarrow E_{n}$ for $m \geq n$ ) and note that $\lim _{\leftarrow} E_{n} \cong$ $E$, so for convenience we write $E=\lim _{\leftarrow} E_{n}$.

For each $X \subseteq E$ and each $n \in \mathbb{N}$, we set $X_{n}=j_{n} \mu_{n}(X)$ and we let $\overline{X_{n}}$ and $\partial X_{n}$ denote, respectively, the closure and the boundary of $X_{n}$ with respect to $|\cdot|_{n}$ in $E_{n}$. Also the pseudointerior of $X$ is defined by [2]

$$
\text { pseudo }-\operatorname{int}(X)=\left\{x \in X: j_{n} \mu_{n}(x) \in \overline{X_{n}} \backslash \partial X_{n} \text { for every } n \in \mathbb{N}\right\}
$$

Our main result in this paper is the extension of Theorem 1.1 to an applicable result in the Fréchet space setting (we refer the reader to [1]; in applications, usually the set $U$ is bounded and as a result has empty interior in the nonnormable situation).

Theorem 2.2. Let $E$ and $E_{n}$ be as described above and let $F: X \rightarrow 2^{E}$, where $X \subseteq E$ (here $2^{E}$ denotes the family of nonempty subsets of $E$ ). Suppose that the following conditions are satisfied:

$$
x_{0} \in \text { pseudo }-\operatorname{int}(X),
$$

for each $n \in \mathbb{N}, \quad F: \overline{X_{n}} \longrightarrow C K\left(E_{n}\right)$ is an upper semicontinuous map,

for each $n \in \mathbb{N}, \quad M \subseteq \overline{X_{n}}$ with $M \subseteq \operatorname{co}\left(\left\{j_{n} \mu_{n}\left(x_{0}\right)\right\} \cup F(M)\right)$,

with $\bar{M}=\bar{C}$ and $C \subseteq M$ countable, implies that $\bar{M}$ is compact

for each $n \in \mathbb{N}, \quad y \notin(1-\lambda) j_{n} \mu_{n}\left(x_{0}\right)+\lambda F y$ in $E_{n} \quad \forall \lambda \in(0,1), y \in \partial X_{n}$,

for each $n \in\{2,3, \ldots\}$ if $y \in \overline{X_{n}}$ solves $y \in F y$ in $E_{n}$, then $y \in \overline{X_{k}}$,

$$
\text { for } k \in\{1, \ldots, n-1\} \text {. }
$$

Then $F$ has a fixed point in X. 
Proof. Fix $n \in \mathbb{N}$. Let $\sum_{n}=\left\{x \in \overline{X_{n}}: x \in F x\right.$ in $\left.E_{n}\right\}$. Now Theorem 1.1 (note that (2.6) implies that $\left.j_{n} \mu_{n}\left(x_{0}\right) \in \overline{X_{n}} \backslash \partial X_{n}\right)$ guarantees that there exists $y_{n} \in \sum_{n}$ with $y_{n} \in F y_{n}$. We look at $\left\{y_{n}\right\}_{n \in \mathbb{N}}$. Now $y_{1} \in \sum_{1}$. Also $y_{k} \in \sum_{1}$ for $k \in \mathbb{N} \backslash\{1\}$ since $y_{k} \in \overline{X_{1}}$ from (2.10) (see also (2.4)). As a result, $y_{n} \in \sum_{1}$ for $n \in \mathbb{N}$ and since $\sum_{1}$ is compact (see Remark 1.2), there exist a subsequence $N_{1}^{\star}$ of $\mathbb{N}$ and a $z_{1} \in \sum_{1}$ with $y_{n} \rightarrow z_{1}$ in $E_{1}$ as $n \rightarrow \infty$ in $N_{1}^{\star}$. Let $N_{1}=N_{1}^{\star} \backslash\{1\}$. Now $y_{n} \in \sum_{2}$ for $n \in N_{1}$ so there exist a subsequence $N_{2}^{\star}$ of $N_{1}$ and a $z_{2} \in \sum_{2}$ with $y_{n} \rightarrow z_{2}$ in $E_{2}$ as $n \rightarrow \infty$ in $N_{2}^{\star}$. Note from (2.4) that $z_{2}=z_{1}$ in $E_{1}$ since $N_{2}^{\star} \subseteq N_{1}$. Let $N_{2}=N_{2}^{\star} \backslash\{2\}$. Proceed inductively to obtain subsequences of integers

$$
N_{1}^{\star} \supseteq N_{2}^{\star} \supseteq \cdots, \quad N_{k}^{\star} \subseteq\{k, k+1, \ldots\}
$$

and $z_{k} \in \sum_{k}$ with $y_{n} \rightarrow z_{k}$ in $E_{k}$ as $n \rightarrow \infty$ in $N_{k}^{\star}$. Note that $z_{k+1}=z_{k}$ in $E_{k}$ for $k \in\{1,2, \ldots\}$. Also let $N_{k}=N_{k}^{\star} \backslash\{k\}$.

Fix $k \in \mathbb{N}$. Let $y=z_{k}$ in $E_{k}$. Notice that $y$ is well defined and $y \in \lim _{\leftarrow} E_{n}=E$. Now $y_{n} \in F y_{n}$ in $E_{n}$ for $n \in N_{k}$ and $y_{n} \rightarrow y$ in $E_{k}$ as $n \rightarrow \infty$ in $N_{k}$ (since $y=z_{k}$ in $E_{k}$ ) together with the fact that $F: \overline{X_{k}} \rightarrow C K\left(E_{k}\right)$ is upper semicontinuous (note that $y_{n} \in \sum_{k}$ for $n \in N_{k}$ ) imply that $y \in F y$ in $E_{k}$. We can do this for each $k \in \mathbb{N}$ so as a result, we have $y \in F y$ in $E$.

Next, we present an application of Theorem 2.2. We discuss the differential equation

$$
\begin{gathered}
y^{\prime}(t)=f(t, y(t)) \quad \text { a.e. } t \in[0, T), \\
y(0)=y_{0} \in \mathbb{R},
\end{gathered}
$$

where $0<T \leq \infty$ is fixed. First we introduce some notation. If $u \in C[0, T)$, then for every $n \in \mathbb{N}$, we define the seminorms $\rho_{n}(u)$ by

$$
\rho_{n}(u)=\sup _{t \in\left[0, t_{n}\right]}|u(t)|
$$

where $t_{n} \uparrow T$. Note that $C[0, T)$ is a locally convex linear topological space. The topology on $C[0, T)$, induced by the seminorms $\left\{\rho_{n}\right\}_{n \in \mathbb{N}}$, is the topology of uniform convergence on every compact interval of $[0, T)$.

Recall that a function $g:[a, b] \times \mathbb{R} \rightarrow \mathbb{R}$ is an $L^{1}$-Carathéodory function if

(a) the map $t \mapsto g(t, y)$ is measurable for all $y \in \mathbb{R}$,

(b) the map $y \mapsto g(t, y)$ is continuous for a.e. $t \in[a, b]$.

Now, $g:[a, b] \times \mathbb{R} \rightarrow \mathbb{R}$ is said to be an $L^{p}$-Carathéodory function $(1 \leq p \leq \infty)$ if $g$ is a Carathéodory function and

(c) for any $r>0$, there exists $\mu_{r} \in L^{p}[a, b]$ such that $|y| \leq r$ implies that $|g(t, y)| \leq$ $\mu_{r}(t)$ for a.e. $t \in[a, b]$.

Finally, a function $g:[0, T) \times \mathbb{R} \rightarrow \mathbb{R}$ is an $L_{\text {loc }}^{p}$-Carathéodory function if (a), (b), and (c) above hold when $g$ is restricted to $\left[0, t_{n}\right] \times \mathbb{R}$ for any $n \in \mathbb{N}$. 
Theorem 2.3. Suppose that the following conditions are satisfied:

$$
\text { for each } n \in \mathbb{N}, f:\left[0, t_{n}\right] \times \mathbb{R} \longrightarrow \mathbb{R} \text { is a Carathéodory function, }
$$

there exists an $L_{\mathrm{loc}}^{1}[0, T)-$ Carathéodory function $g:[0, T) \times[0, \infty) \longrightarrow[0, \infty)$

such that $|f(t, x)| \leq g(t,|x|) \quad$ for a.e. $t \in[0, T)$ and all $x \in \mathbb{R}$,

for each $n \in \mathbb{N}$, the problem

$$
\begin{gathered}
v^{\prime}(t)=g(t, v(t)), \quad \text { a.e. } t \in\left[0, t_{n}\right], \\
v(0)=\left|y_{0}\right|
\end{gathered}
$$

has a maximal solution $r_{n}(t)$ on $\left[0, t_{n}\right]\left(\right.$ here $\left.r_{n} \in C\left[0, t_{n}\right]\right)$.

Then (2.12) has at least one solution $y \in C[0, T)$.

Remark 2.4. One could also obtain a multivalued version of Theorem 2.3 (with (2.12) replaced by a differential inclusion) by using the ideas in the proof below with the ideas in [6].

Proof. Here $E=C[0, T), \mathbf{E}^{k}$ consists of the class of functions in $E$ which coincide on the interval $\left[0, t_{k}\right], E_{k}=C\left[0, t_{k}\right]$ with of course $\pi_{n, m}=j_{n} \mu_{n, m} j_{m}^{-1}: E_{m} \rightarrow E_{n}$ for $m \geq n$ defined by $\pi_{n, m}(x)=\left.x\right|_{\left[0, t_{n}\right]}$. We will apply Theorem 2.2 with

$$
X=\left\{u \in C[0, T):|u|_{n} \leq w_{n} \text { for each } n \in \mathbb{N}\right\}
$$

here $|u|_{n}=\sup _{t \in I_{n}}|u(t)|$, where $I_{n}=\left[0, t_{n}\right]$ and $w_{n}=\sup _{t \in I_{n}} r_{n}(t)+1$. On any interval $I_{n}=\left[0, t_{n}\right](n \in \mathbb{N})$, we let $F$ on $C\left(I_{n}\right)$ be defined by

$$
F y(t)=y_{0}+\int_{0}^{t} f(s, y(s)) d s
$$

Fix $n \in \mathbb{N}$. Notice that

$$
\overline{X_{n}}=\left\{u \in C\left[0, t_{n}\right]:|u|_{n} \leq w_{n}\right\}
$$

Clearly, (2.6) holds with $x_{0}=0$ and a standard argument from the literature [8] guarantees that

$$
F: \overline{X_{n}} \longrightarrow E_{n} \text { is continuous and compact, }
$$

so (2.7) and (2.8) hold.

To show that (2.9), fix $n \in \mathbb{N}$ and let $y \in C\left(I_{n}\right)$ be such that $y=\lambda F y$ for $\lambda \in(0,1)$. We claim $|y|_{n}<w_{n}$ and if this is true, then $y \notin \partial X_{n}$ and hence (2.9) is true. Let $t \in I_{n}$ and we now show that $|y(t)|<w_{n}$. If $|y(t)| \leq\left|y_{0}\right|$, we are finished so it remains to discuss the 
case when $|y(t)|>\left|y_{0}\right|$. In this case, there exists $a \in[0, t)$ with

$$
|y(s)|>\left|y_{0}\right| \quad \text { for } s \in(a, t],|y(a)|=\left|y_{0}\right| .
$$

Also

$$
|y(s)|^{\prime} \leq\left|y^{\prime}(s)\right| \leq g(s,|y(s)|) \quad \text { a.e. on }(a, t)
$$

so

$$
\begin{gathered}
|y(s)|^{\prime} \leq g(s,|y(s)|), \quad \text { a.e. on }(a, t), \\
|y(a)|=\left|y_{0}\right| .
\end{gathered}
$$

Now a standard comparison theorem for ordinary differential equations in the real case [5, Theorem 1.10.2] guarantees that $|y(s)| \leq r_{n}(s)$ for $s \in[a, t]$, so in particular $|y(t)| \leq$ $r_{n}(t)<w_{n}$, so $(2.9)$ is true.

It remains to show that (2.10). To see this, fix $n \in\{2,3, \ldots\}$ and suppose that $y \in \overline{X_{n}}$ solves

$$
\begin{gathered}
y^{\prime}(t)=f(t, y(t)), \quad \text { a.e. on }\left[0, t_{n}\right], \\
y(0)=y_{0} .
\end{gathered}
$$

Next, fix $k \in\{1, \ldots, n-1\}$. We must show that $y \in \overline{X_{k}}$. Now since $t_{n} \uparrow T$, notice that $\left[0, t_{k}\right] \subseteq\left[0, t_{n}\right]$ so as a result,

$$
\begin{gathered}
y^{\prime}(t)=f(t, y(t)), \quad \text { a.e. on }\left[0, t_{k}\right], \\
y(0)=y_{0} .
\end{gathered}
$$

Let $t \in\left[0, t_{k}\right]$ and essentially the same argument as above guarantees that $|y(t)|<w_{k}$ so $|y|_{k}<w_{k}$. Thus $y \in \overline{X_{k}}$ and (2.10) holds.

The result now follows immediately from Theorem 2.2 .

Our final result was motivated by Urysohn-type operators.

Theorem 2.5. Let $E$ and $E_{n}$ be as described in the beginning of Section 2 and let $F: X \rightarrow 2^{E}$, where $X \subseteq E$. Suppose that the following conditions are satisfied:

$$
\begin{gathered}
x_{0} \in \text { pseudo }-\operatorname{int}(X), \\
\overline{X_{1}} \supseteq \overline{X_{2}} \supseteq \cdots,
\end{gathered}
$$

for each $n \in \mathbb{N}, \quad F_{n}: \overline{X_{n}} \longrightarrow C K\left(E_{n}\right)$ is upper semicontinuous, for each $n \in \mathbb{N}, \quad M \subseteq \overline{X_{n}} \quad$ with $M \subseteq \operatorname{co}\left(\left\{j_{n} \mu_{n}\left(x_{0}\right)\right\} \cup F_{n}(M)\right)$ with $\bar{M}=\bar{C}$ and $C \subseteq M \quad$ countable, implies that $\bar{M}$ is compact,

for each $n \in \mathbb{N}, \quad y \notin(1-\lambda) j_{n} \mu_{n}\left(x_{0}\right)+\lambda F_{n} y$ in $E_{n} \quad \forall \lambda \in(0,1), y \in \partial X_{n}$, 
for each $n \in \mathbb{N}$, the map $\mathcal{K}_{n}: \overline{X_{n}} \longrightarrow 2^{E_{n}}$, given by

$$
\mathscr{K}_{n}(y)=\bigcup_{m=n}^{\infty} F_{m}(y) \text { (see Remark 2.6), satisfies that }
$$

if $C \subseteq \overline{X_{n}}$ is countable with $C \subseteq \mathscr{K}_{n}(C)$, then $\bar{C}$ is compact,

if there exist $a w \in X$ and a sequence $\left\{y_{n}\right\}_{n \in \mathbb{N}}$ with $y_{n} \in \overline{X_{n}}$ and $y_{n} \in F_{n} y_{n}$ in $E_{n}$

such that for every $k \in \mathbb{N}$ there exists a subsequence $S \subseteq\{k+1, k+2, \ldots\}$ of $\mathbb{N}$ with $y_{n} \longrightarrow w$ in $E_{k}$ as $n \longrightarrow \infty$ in $S$, then $w \in F w$ in $E$.

Then $F$ has a fixed point in $X$.

Remark 2.6. The definition of $\mathscr{K}_{n}$ is as follows. If $y \in \overline{X_{n}}$ and $y \notin \overline{X_{n+1}}$, then $\mathcal{K}_{n}(y)=$ $F_{n}(y)$, whereas if $y \in \overline{X_{n+1}}$ and $y \notin \overline{X_{n+2}}$, then $\mathcal{K}_{n}(y)=F_{n}(y) \cup F_{n+1}(y)$, and so on.

Proof. Fix $n \in \mathbb{N}$. Let $\sum_{n}=\left\{x \in \overline{X_{n}}: x \in F_{n} x\right.$ in $\left.E_{n}\right\}$. Now, Theorem 1.1 guarantees that there exists $y_{n} \in \sum_{n}$ with $y_{n} \in F_{n} y_{n}$ in $E_{n}$. We look at $\left\{y_{n}\right\}_{n \in \mathbb{N}}$. Note that $y_{n} \in \overline{X_{1}}$ for $n \in$ $\mathbb{N}$ from (2.27). In addition with $C=\left\{y_{n}\right\}_{1}^{\infty}$, we have from assumption (2.31) that $\bar{C}\left(\subseteq E_{1}\right)$ is compact; note that $y_{n} \in \mathscr{K}_{1}\left(y_{n}\right)$ in $E_{1}$ for each $n \in \mathbb{N}$. Thus there exist a subsequence $N_{1}^{\star}$ of $\mathbb{N}$ and a $z_{1} \in \overline{X_{1}}$ with $y_{n} \rightarrow z_{1}$ in $E_{1}$ as $n \rightarrow \infty$ in $N_{1}^{\star}$. Let $N_{1}=N_{1}^{\star} \backslash\{1\}$. Proceed inductively to obtain subsequences of integers

$$
N_{1}^{\star} \supseteq N_{2}^{\star} \supseteq \ldots, \quad N_{k}^{\star} \subseteq\{k, k+1, \ldots\}
$$

and $z_{k} \in \overline{X_{k}}$ with $y_{n} \rightarrow z_{k}$ in $E_{k}$ as $n \rightarrow \infty$ in $N_{k}^{\star}$. Note that $z_{k+1}=z_{k}$ in $E_{k}$ for $k \in \mathbb{N}$. Also let $N_{k}=N_{k}^{\star} \backslash\{k\}$.

Fix $k \in \mathbb{N}$. Let $y=z_{k}$ in $E_{k}$. Notice that $y$ is well defined and $y \in \lim _{\leftarrow} E_{n}=E$. Now $y_{n} \in F_{n} y_{n}$ in $E_{n}$ for $n \in N_{k}$ and $y_{n} \rightarrow y$ in $E_{k}$ as $n \rightarrow \infty$ in $N_{k}$ (since $y=z_{k}$ in $E_{k}$ ) together with (2.32) imply that $y \in F y$ in $E$.

\section{References}

[1] R. P. Agarwal, M. Frigon, and D. O'Regan, A survey of recent fixed point theory in Fréchet spaces, Nonlinear Analysis and Applications: to V. Lakshmikantham on His 80th Birthday. Vol. 1, 2, Kluwer Academic, Dordrecht, 2003, pp. 75-88.

[2] M. Frigon, Fixed point results for compact maps on closed subsets of Fréchet spaces and applications to differential and integral equations, Bull. Belg. Math. Soc. Simon Stevin 9 (2002), no. $1,23-37$.

[3] M. Frigon and D. O'Regan, A Leray-Schauder alternative for Mönch maps on closed subsets of Fréchet spaces, Z. Anal. Anwendungen 21 (2002), no. 3, 753-760.

[4] L. V. Kantorovich and G. P. Akilov, Functional Analysis in Normed Spaces, International Series of Monographs in Pure and Applied Mathematics, vol. 46, The Macmillan, New York, 1964.

[5] V. Lakshmikantham and S. Leela, Differential and Integral Inequalities: Theory and Applications. Vol. I: Ordinary Differential Equations, Mathematics in Science and Engineering, vol. 55, Academic Press, New York, 1969.

[6] D. O'Regan, Maximal solutions and multivalued differential and integral inclusions on a noncompact interval, to appear in Nonlinear Funct. Anal. Appl. 


\section{Fixed point theory}

[7] D. O'Regan and R. P. Agarwal, Fixed point theory for admissible multimaps defined on closed subsets of Fréchet spaces, J. Math. Anal. Appl. 277 (2003), no. 2, 438-445.

[8] D. O'Regan and M. Meehan, Existence Theory for Nonlinear Integral and Integrodifferential Equations, Mathematics and Its Applications, vol. 445, Kluwer Academic, Dordrecht, 1998.

[9] D. O'Regan and R. Precup, Fixed point theorems for set-valued maps and existence principles for integral inclusions, J. Math. Anal. Appl. 245 (2000), no. 2, 594-612.

Ravi P. Agarwal: Department of Mathematical Sciences, College of Science, Florida Institute of Technology, Melbourne, FL 32901-6975, USA

E-mail address: agarwal@fit.edu

Jewgeni H. Dshalalow: Department of Mathematical Sciences, College of Science, Florida Institute of Technology, Melbourne, FL 32901-6975, USA

E-mail address: eugene@winnie.fit.edu

Donal O’Regan: Department of Mathematics, National University of Ireland, Galway, University Road, Galway, Ireland

E-mail address: donal.oregan@nuigalway.ie 


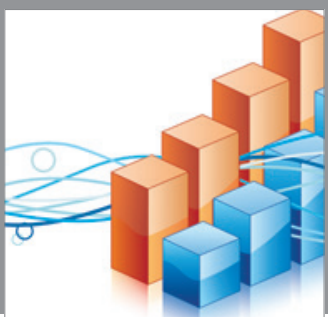

Advances in

Operations Research

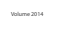

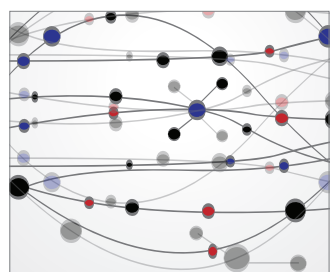

\section{The Scientific} World Journal
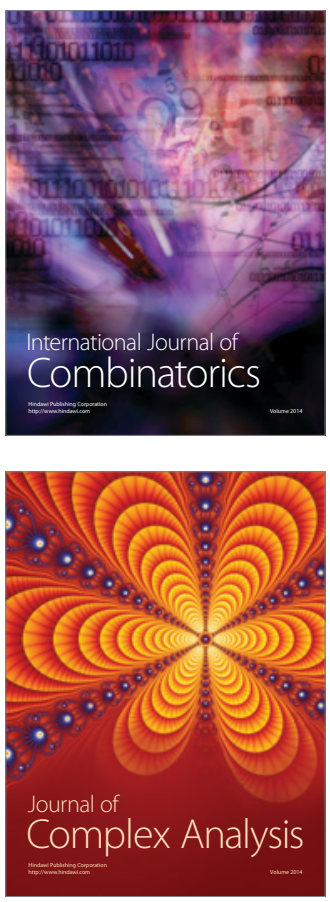

International Journal of

Mathematics and

Mathematical

Sciences
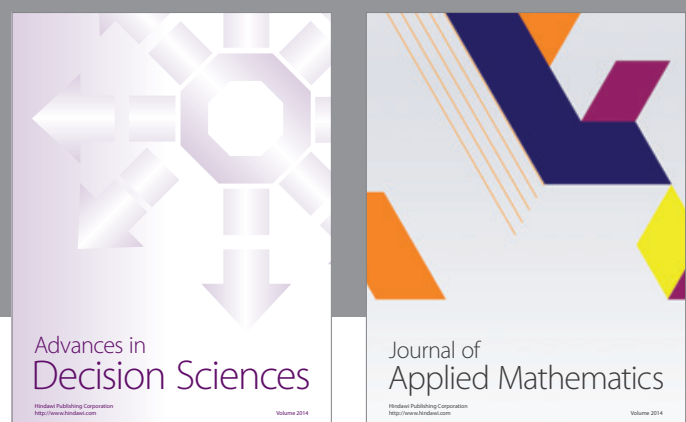

Journal of

Applied Mathematics
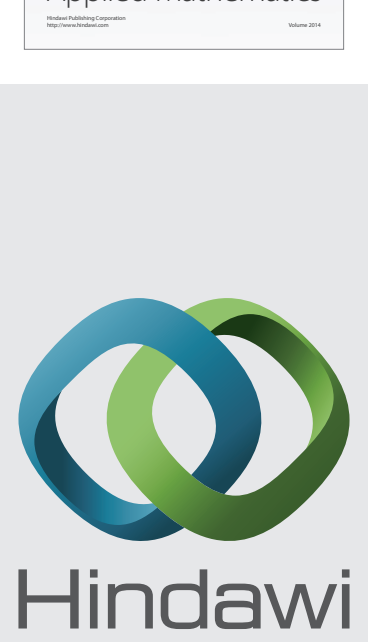

Submit your manuscripts at http://www.hindawi.com
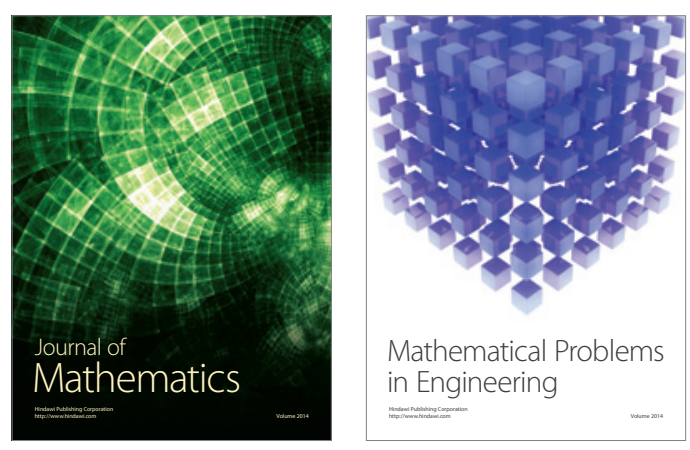

Mathematical Problems in Engineering
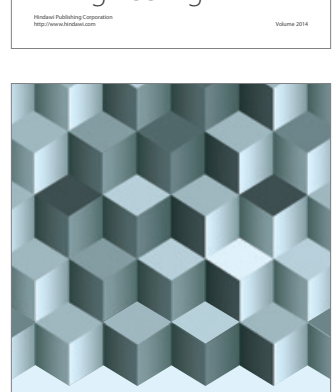

Journal of

Function Spaces
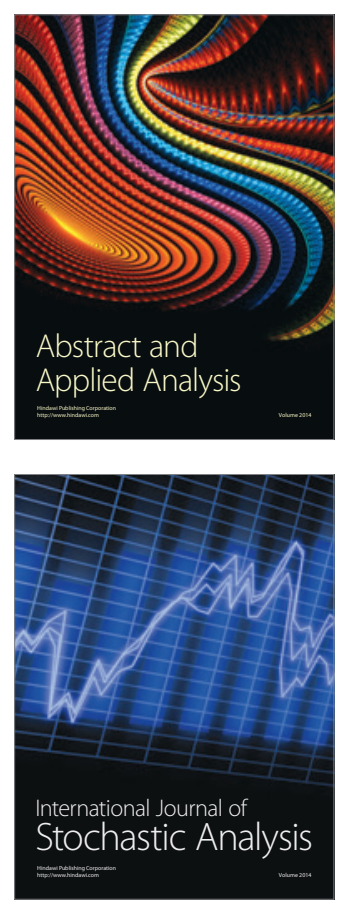

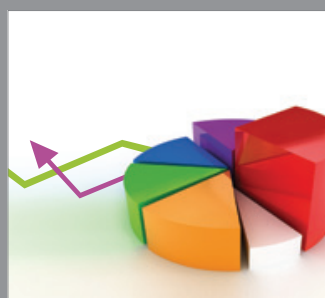

ournal of

Probability and Statistics

Promensencen
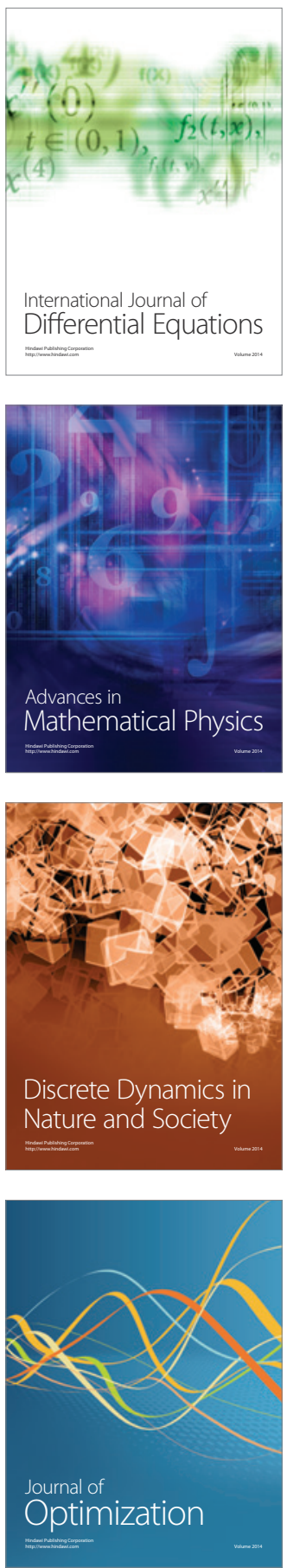